\title{
Positronium Confinement in Small Cavities: A Two-Particle Model for the Lowering of Contact Density
}

\author{
G. Marlotti Tanzi, ${ }^{1}$ F. Castelli, ${ }^{1,2^{*}}$ and G. Consolati ${ }^{3,2}$ \\ ${ }^{1}$ Department of Physics, Università degli Studi di Milano, via Celoria 16, I-20133 Milano, Italy \\ ${ }^{2}$ INFN, sezione di Milano, via Celoria 16, I-20133 Milano, Italy \\ ${ }^{3}$ Department of Aerospace Science and Technology, Politecnico di Milano, via LaMasa 34, I-20156 Milano, Italy
}

(Received 20 May 2015; published 22 January 2016)

\begin{abstract}
Positronium (Ps) is widely used as a probe for studying nanometric porosities in condensed matter. Accessible experimental measurements concern annihilation rates by pickoff processes and contact densities (the electron density at the positron position). Existing models for describing Ps properties in small cavities do not justify the lowering of the contact density with respect to that of Ps in vacuum, as found in most materials. We formulate a two-particle model in which only the electron is confined in the cavity, while the positron is moving freely and feels the medium via a positive work function. Our calculation fully explains experimental data for a large class of materials and suggests a way to gain information on pore sizes and positron work functions.
\end{abstract}

DOI: 10.1103/PhysRevLett.116.033401

Positronium (Ps) is a hydrogenlike bound state of an electron and its antiparticle, the positron. Ps can exist in two different states: the singlet state $p$-Ps, rapidly decaying into $2 \gamma$ rays with a lifetime of $0.125 \mathrm{~ns}$, and the triplet state $o$-Ps annihilating in $3 \gamma$ rays with a lifetime of $142 \mathrm{~ns}$ in vacuum [1]. Ps is usually formed by implanting positrons in condensed matter. Many insulators and molecular solids allow Ps formation, sometimes with high efficiency; when generated near the surface (e.g., by using a slow positron beam) Ps can rapidly spread toward the surface and eventually be released into the vacuum [2]. In porous materials, $o$-Ps is also emitted into cavities, and, mainly in subnanometricsized voids, its lifetime can be notably reduced by pickoff annihilation: the $o$-Ps positron can annihilate via $2 \gamma$ decay with an electron of the surrounding cavity walls if a relative singlet state is realized [3]. This property is at the heart of the use of Ps as a probe for studying nanoscale structures in condensed matter. In fact, while $2 \gamma$ annihilation in vacuum depends on the electron density at the positron position (the contact density [4]), pickoff processes depend on the electron density in the bulk around the cavity.

Experimental data obtained with the positron annihilation lifetime spectroscopy technique, also in the presence of a magnetic field (via magnetic quenching), primarily concern pickoff annihilation with lifetimes as low as a few ns $[5,6]$. The contact density can be determined from fitting lifetime spectra [7]. In molecular solids, this quantity is usually found to be well below the vacuum value. It is of paramount importance to connect these measurable quantities with the properties of Ps in small cavities as a means to obtain information on pore dimensions and other material characteristics.

To describe Ps inside small cavities, the most used models are based on the Tao-Eldrup approach [8-11], which relates pickoff annihilation rates to pore sizes by considering Ps as a single quantum particle. Consequently, these models cannot give information about contact density and are suitable only when cavities are much larger than twice the Ps Bohr radius $\alpha_{0}=0.106 \mathrm{~nm}$. Another family of models, more appropriate for subnanometer cavities, consider both Ps constituent particles as independent but confined into the cavity $[12,13]$ or interacting with the material through effective potentials [14]. A significant increase in energy and contact density is found for cavities with radius smaller than $0.5 \mathrm{~nm}$, contrary to the experimental evidence. A different approach is considered in Ref. [15]: the electron-positron pair bounded by Coulombic interaction forms a bubblelike state in a dielectric medium. A contact density lowering is demonstrated, but the results are not easily applicable to real materials due to special assumptions about work functions.

Anyway, these models are not fully satisfactory and fail to give a clear physical picture of the lowering of the contact density for the following reason. Even if the presence of a confining potential on the Ps atom, as a whole, is well accepted and proven by successful predictions of annihilation spectra, our concern is that this potential is a net result of two independent and different contributions acting on the electron and on the positron separately. Because of the complex interactions with bulk electrons, it seems unlikely that the positron remains confined into a cavity. A positive value for the positron work function in molecular solids and semiconductors is derived by theoretical models describing positrons in condensed matter [16-18] and is found, for example, in silica [19]. This leads to an effective attraction of the positron toward the medium. On the other hand, a confining effect must act on the electron paired with the positron. This 
is the potential postulated in the Tao-Eldrup model $[8,9]$, and its origin may be traced in the strong repulsive Pauli exchange forces with bulk electrons, which eventually dominates the effect of the electron work function.

Starting from these observations, we reconsider the problem of a Ps atom in nanopores by formulating a two-particle model with appropriate potentials, different for each particle. Assuming for definiteness a spherical cavity of radius $R_{c}$ centered on the axes origin, and using relative $\vec{r}$ and center-of-mass $\vec{R}$ coordinates, the Hamiltonian of the positron electron system reads

$$
H=-\frac{\hbar^{2}}{4 m} \nabla_{R}^{2}-\frac{\hbar^{2}}{m} \nabla_{r}^{2}-\frac{e^{2}}{|\vec{r}|}+V_{\text {conf }}+V_{\text {bulk }},
$$

where $m$ is the electron or positron mass. $V_{\text {conf }}$ and $V_{\text {bulk }}$ describe the interaction with the surrounding medium. The confining potential acts only on the electron and can be taken as a well with infinite depth:

$$
V_{\text {conf }}(\vec{r}, \vec{R})=\left\{\begin{array}{ll}
0 & \text { if }\left|\vec{r}_{-}\right|<R_{c} \\
\infty & \text { if }\left|\vec{r}_{-}\right|>R_{c}
\end{array},\right.
$$

where $\vec{r}_{-}=\vec{R}-\vec{r} / 2$ is the electron position. This defines the geometrical domain $\Omega(\vec{r}, \vec{R})$, out of which the twoparticle wave function is zero [i.e., $\Psi(\vec{r}, \vec{R})=0$ if $\vec{R}$, $\vec{r} \notin \Omega(\vec{r}, \vec{R})]$. The bulk potential acts only on the positron. In the first approximation, it can be taken as equal to the opposite of the work function deep inside the bulk and zero in the electron-confining cavity. We further consider the presence of a transition surface layer of thickness $\Delta s$ representing the offset between the outermost position accessible to the electron and the region where positron bulk properties become predominant. This layer is similar to that introduced in the Tao-Eldrup model to justify pickoff processes between a confined Ps and the local electron density. A simple explicit form of the potential is then

$$
V_{\text {bulk }}(\vec{r}, \vec{R})= \begin{cases}0 & \text { if }\left|\vec{r}_{+}\right|<R_{c}+\Delta s \\ -\phi_{+} & \text {if }\left|\vec{r}_{+}\right|>R_{c}+\Delta s\end{cases}
$$

where $\vec{r}_{+}=\vec{R}+\vec{r} / 2$ is the positron position, and $\phi_{+}$is the (positive) work function. This potential model is also a generalization of what is found with density-functional theory in metals [18]. Typical values of $\Delta s$ are in the range $0.1-0.2 \mathrm{~nm}$, of the order of the bond length between atoms. As a matter of fact, different reasonable choices of $\Delta s$ have negligible effects on our results; hence, following Ref. [8], we fix this parameter at $\Delta s=0.17 \mathrm{~nm}$ (see Fig. 3).

The calculation of the contact density requires the knowledge of the two-particle wave function in $\vec{r}=0$, irrespective of the center-of-mass position $\vec{R}$. We look for a factorized solution of the Schrödinger equation. Noting that the center of mass is not confined and can be also located outside the cavity $\left(R>R_{c}\right)$, as long as both $\vec{R}, \vec{r} \in \Omega(\vec{R}, \vec{r})$, a convenient choice for its representation, not requiring the introduction of additional geometrical parameters, is a plane-wave-like factor. The confinement effects are taken into account by means of the relative-motion part $\tilde{\varphi}$ of the two-particle wave function:

$$
\Psi(\vec{r}, \vec{R}) \simeq \frac{1}{\sqrt{\tilde{V}}} e^{i \vec{K} \cdot \vec{R}} \tilde{\varphi}(\vec{r}, R) \quad(\vec{R}, \vec{r} \in \Omega(\vec{r}, \vec{R})),
$$

where $\tilde{V}$ is a suitable normalization volume. In the spherically symmetric cavity, the function $\tilde{\varphi}(\vec{r}, R)$ depends parametrically on $R$, the distance of the center of mass from the origin, and retains the coupling between the relative and the center-of-mass coordinates via the geometrical constraint $\Omega(\vec{r}, \vec{R})$. It can be constructed as a piecewise continuous function on different $R$ intervals, as described in the following.

If $R$ lies deep inside the cavity, we expect that this function resembles the (normalized) ground-state vacuum solution: $\tilde{\varphi}(\vec{r}, R \rightarrow 0) \simeq \varphi_{0}(r, \theta, \phi)=(2 / \sqrt{4 \pi}) \alpha_{0}^{-3 / 2} e^{-r / \alpha_{0}}$. As $R$ approaches the cavity wall, it will slowly change toward an outermost function $\tilde{\varphi}\left(\vec{r}, R \sim R_{c}\right) \simeq \varphi_{\text {wall }}(\vec{r})$, strongly deformed with respect to the simpler spherical one due to the constraint on the electron position. To find an expression for $\varphi_{\text {wall }}(\vec{r})$, let us first consider the special case in which the electron is located just near the wall $\left(\left|\vec{r}_{-}\right| \simeq R_{c}\right)$. Denoting with $\vec{r}_{p}$ the positron position, an equation for the spherically symmetric radial ground-state $(l=0)$ wave function describing the positron in the medium can be derived from Eqs. (1) and (3) [20]:

$$
\begin{aligned}
- & \frac{\hbar^{2}}{m}\left[u^{\prime \prime}\left(r_{p}\right)+\frac{2}{r} u^{\prime}\left(r_{p}\right)\right]-\left(\frac{e^{2}}{r_{p}}+\phi_{+} \Theta\left(r_{p}-\Delta s\right)\right) u\left(r_{p}\right) \\
& =E u\left(r_{p}\right) .
\end{aligned}
$$

An example of a solution for the positron radial probability distribution $r_{p}^{2} u^{2}\left(r_{p}\right)$ is plotted in Fig. 1 showing the wave function polarization toward the bulk due to the positive work function. Now, focusing on the cavity wall around the fixed electron position, and considering a reference system in the tangent plane having a $z$ axis pointing outside the cavity, the $\varphi_{\text {wall }}(\vec{r})$ can be built as a superposition between the inner free Ps wave function and the outer one $\varphi_{\text {ext }}(r, \theta, \phi) \equiv u\left(r_{p}=r\right)$ :

$$
\varphi_{\text {wall }}(\vec{r}) \simeq \begin{cases}A \varphi_{\text {ext }}(r, \theta, \phi) & \text { if } 0<\theta<\pi / 2 \\ B \varphi_{0}(r, \theta, \phi) & \text { if } \pi / 2<\theta<\pi\end{cases}
$$

Since both $\varphi_{\mathrm{ext}}$ and $\varphi_{0}$ are normalized over the entire space, the normalization of $\varphi_{\text {wall }}$ implies that the coefficients $A$ and $B$ must satisfy the condition $A^{2} / 2+B^{2} / 2=1$. Another constraint derives from the need of a unique value 


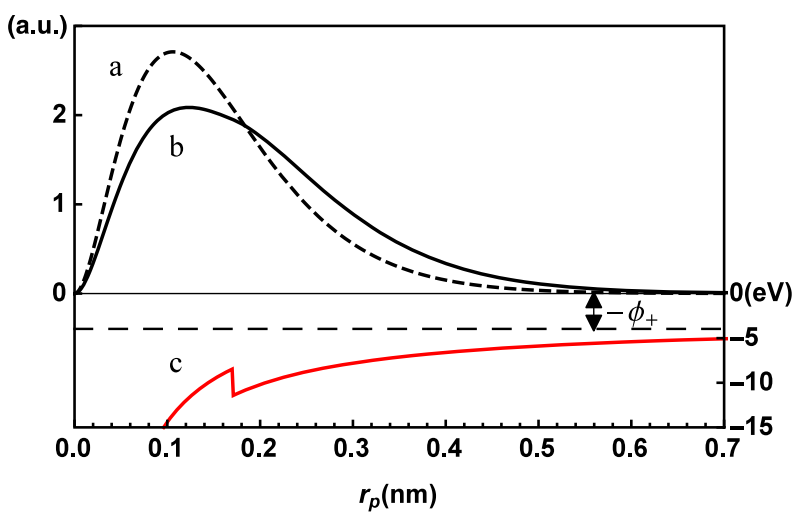

FIG. 1. Positron radial probability distribution (arbitrary units) in free space (a) and inside a medium with work function $\phi_{+}=$ $3 \mathrm{eV}$ (b). The electron is in $r_{p}=0$ (at the cavity wall), and the surface layer is placed at $\Delta s=0.17 \mathrm{~nm}$. Line c (red) represents the total potential acting on the positron.

of the wave function in $r=0: \varphi_{\text {wall }}(0)=\lim _{r \rightarrow 0} A \varphi_{\text {ext }}(r)=$ $\lim _{r \rightarrow 0} B \varphi_{0}(r)$. Hence, these coefficients are determined as functions of $\phi_{+}$and $\Delta s$. As expected, $A>1$ and $B<1$ due to the stretching of the external part of the wave function toward the medium, which, near the wall, determines a lower value with respect to that in vacuum.

Finally, a simple piecewise representation for $\tilde{\varphi}(\vec{r}, R)$ can be built, without lacking generality, if we admit that the bulk polarization effects are important only within a shell of thickness $\alpha_{0}$ (the Ps Bohr radius) below the cavity wall, because it is unlikely that the wave function polarization effect operates over a larger range. Hence,

$$
\tilde{\varphi}(\vec{r}, R) \simeq \begin{cases}\varphi_{0}(r, \theta, \phi) & \text { if } 0 \leq R<R_{c}-\alpha_{0} \\ \varphi_{\text {wall }}(r, \theta, \phi) & \text { if } R \geq R_{c}-\alpha_{0}\end{cases}
$$

We now have all the elements for the calculation of the Ps contact density $\kappa$. With the wave function of Eq. (4),

$$
\begin{aligned}
\kappa & =\int_{\Omega(\vec{r}, \vec{R})}\left|\frac{1}{\sqrt{\tilde{V}}} e^{i \vec{K} \cdot \vec{R}} \tilde{\varphi}(\vec{r}, R)\right|^{2} \delta(\vec{r}) d^{3} \vec{R} d^{3} \vec{r} \\
& =\frac{4 \pi}{\tilde{V}} \int_{0}^{R_{c}} \tilde{\kappa}(R) R^{2} d R,
\end{aligned}
$$

where $\tilde{\kappa}(R)=|\tilde{\varphi}(0, R)|^{2}$. Using Eq. (7) one has

$$
\tilde{\kappa}(R)= \begin{cases}\kappa_{0} & \text { if } 0<R \leq R_{c}-\alpha_{0} \\ B^{2} \kappa_{0} & \text { if } R_{c}-\alpha_{0}<R \leq R_{c},\end{cases}
$$

and finally $\kappa=\kappa_{0}(4 \pi / 3 \tilde{V})\left[\left(1-B^{2}\right)\left(R_{c}-\alpha_{0}\right)^{3}+B^{2} R_{c}^{3}\right]$, where $\kappa_{0}=1 / \pi \alpha_{0}^{3}$ is the free Ps contact density.

Given that $B<1$, it is clear that the function $\tilde{\kappa}(R)$ constitutes a first contribution to the lowering of the contact density below the free value. This is a consequence of the modified Ps radial wave function when the electron is near the cavity wall, while the positron can explore the external region. A second contribution comes from the possibility of the center of mass to stand outside the cavity, with a reduced probability of finding the positron inside. This information is contained in the normalization volume $\tilde{V}$ of the Ps wave function:

$$
\tilde{V}=4 \pi \int_{0}^{\infty} d R R^{2} P_{[-]}(R),
$$

where $P_{[-]}(R)$ corresponds to the conditional probability of having the electron confined and the positron both inside or outside the cavity, given the center of mass at distance $R$ from the origin. In Fig. 2, we plot this function comparing a case in which the positron interacts with the external medium, with the case of a free positron (i.e., with null work function). At the cavity wall, a positive positron work function increases $P_{[-]}$over the unperturbed value 0.5 .

Experimental data for Ps in small cavities are usually expressed by means of the relative contact density $\kappa_{r}=\kappa / \kappa_{0}$. In Fig. 3, we show $\kappa_{r}$ as a function of the cavity radius for some values of the positron work function. If $\phi_{+}=0 \mathrm{eV}$, we obtain $\kappa_{r} \simeq 1$, which is the same result implicit in the Tao-Eldrup approach. The maximum value $\phi_{+}=6.8 \mathrm{eV}$ corresponds to the free Ps ionization energy. Note that for a cavity radius larger than a few $\mathrm{nm}$, and for reasonable low values for $\phi_{+}$, the lowering of the contact density becomes negligible.

Another independent measurable quantity is the rate of pickoff annihilation $\lambda_{\mathrm{PO}}$, well studied by many authors $[3,6,8,12,15,21]$. The annihilation rate of $o$-Ps in a small cavity is largely dominated by pickoff and can be expressed as

$$
\lambda_{o-\mathrm{Ps}} \approx \lambda_{\mathrm{PO}}=\pi r_{0}^{2} c \rho_{-} \int_{V_{\mathrm{ext}}}\left|\Psi_{+}\left(\vec{r}_{+}\right)\right|^{2} d^{3} \vec{r}_{+},
$$

where $\rho_{-}$is the bulk electron density (assumed uniform), $r_{0}=2.8 \times 10^{-13} \mathrm{~cm}$ is the classical electron radius, $\Psi_{+}\left(\vec{r}_{+}\right)$is the positron wave function, and the integral is

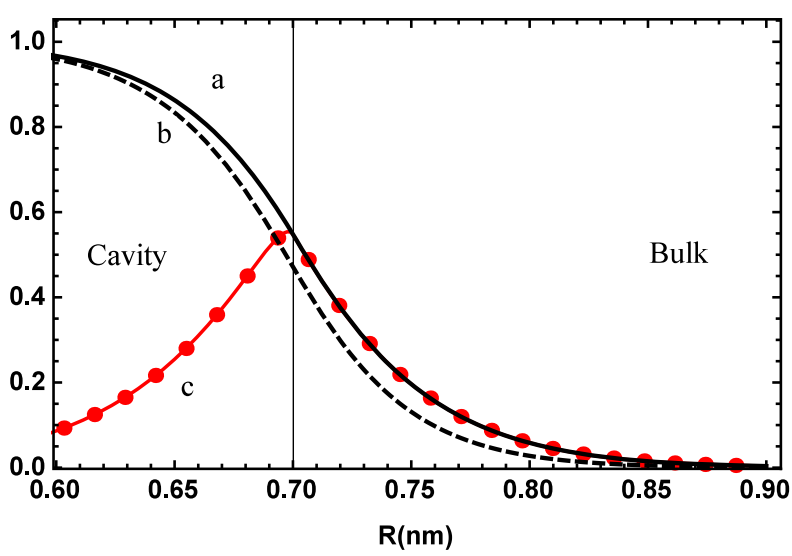

FIG. 2. The function $P_{[-]}(R)$ for a cavity of radius $R_{c}=0.7 \mathrm{~nm}$ and positron work function $\phi_{+}=3 \mathrm{eV}$ (a, full line) or $\phi_{+}=$ $0 \mathrm{eV}$ (b, dashed line). The line $\mathrm{c}$ (red) is the probability distribution $p_{[-\mid+]}(R)$ of Eq. (12). 


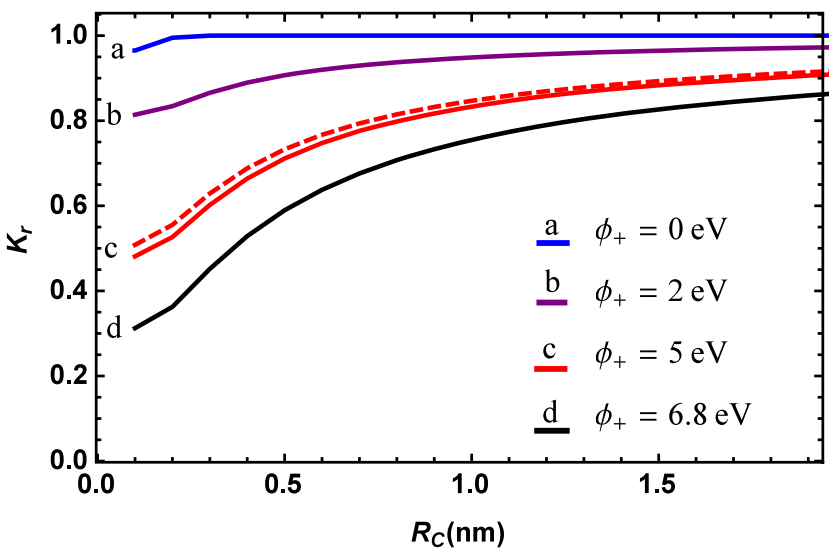

FIG. 3. Ps relative contact density $\kappa_{r}$ as a function of the cavity radius for some values of $\phi_{+}$. Full lines are calculated with the standard choice $\Delta s=0.17 \mathrm{~nm}$; the (red) dashed line is the same as line c but with $\Delta s=0.15 \mathrm{~nm}$, showing that a precise value for this parameter has a negligible effect on contact density.

on the volume $V_{\text {ext }}$, external to the cavity. In the framework of our model, the positron wave function square modulus is obtained by integrating over $\vec{R}$ the expression $|\tilde{\varphi}(\vec{r}, R)|^{2}$, with the electron-confining constraint $\left|\vec{r}_{-}\right|<R_{c}$; in addition, $V_{\text {ext }}$ imposes the condition $\left|\vec{r}_{+}\right|>R_{c}$ [22]. The annihilation rate then results in

$$
\lambda_{\mathrm{PO}}=\frac{4 \pi^{2} r_{0}^{2} c \rho_{-}}{\tilde{V}} \int_{0}^{\infty} d R R^{2} p_{[-\mid+]}(R),
$$

where $p_{[-\mid+]}(R)$ is the conditional probability of having the electron confined and the positron outside the cavity. A plot of this function is shown in Fig. 2.

To complete the calculation of $\lambda_{\mathrm{PO}}$, it is necessary to evaluate the effective electron density $\rho_{-}$interacting with the positron. As a matter of fact, this quantity is extremely hard to estimate because it has a very specific dependence on the physical properties of the material surrounding the cavity. Following the analysis carried out in Ref. [23], the positron "samples" only electrons in the outer atomic shells, essentially $s$ and $p$ electrons in molecular solids. These electrons can be roughly thought of as uniformly spread out over the molecular volume. A suitable approximate expression for molecular volumes can be obtained by considering each constituent atom as a sphere defined by the so-called van der Waals radius [24]. In Table I we report data on van der Waals radii for relevant atomic elements, together with chemical formulas and calculated electron densities for some molecular solids of interest.

To compare in a clearer way experimental data on $o$-Ps contact densities and pickoff lifetimes $\tau_{\mathrm{PO}}=1 / \lambda_{\mathrm{PO}}$ with the corresponding quantities calculated with our model, it is convenient to normalize this lifetime with respect to the electron density by defining the quantity $\tau^{\prime}=\tau_{\mathrm{PO}} \rho_{-} / \kappa_{0}$. It only depends on the cavity radius $R_{c}$ and on the positron work function $\phi_{+}$via the probability $p_{[-\mid+]}(R)$. In Fig. 4, we plot some curves joining the points corresponding to calculated values of $\kappa_{r}$ and $\tau^{\prime}$ for a fixed $\phi_{+}$and different $R_{c}$ (starting from the reasonable minimum value $R_{c}=0.1 \mathrm{~nm}$ ). The known experimental data for some materials taken from Table I are indicated by markers. It must be noted that this kind of representation is the better choice to compare theoretical and experimental data, because it is very difficult to gain independent information on the positron work function and on the pore sizes for most materials.

The general trend shows a lowering of the contact density and of the $o$-Ps lifetime for smaller cavities, as expected. There is a good agreement between our theory and experimental data for a large group of hydrocarbon molecular solids. On the other hand, some compounds stand below the line $\phi_{+}=6.8 \mathrm{eV}$, probably because their effective electron density is lower than our estimation so that their position in the picture should be shifted to the left. An interesting material is silica. In the crystalline form, Ps has a very short lifetime and a very low $\kappa_{r}$. It is expected that no free spaces exist; hence, Ps is delocalized and

TABLE I. Experimental data on relative contact density $\kappa_{r}$ and pickoff lifetime $\tau_{\mathrm{PO}}$ for some selected molecular solids. The column $\tau^{\prime}$ reports the lifetime normalized with respect to the effective electron density $\rho_{-}$calculated using the atomic van der Waals radii (see text) taken from Ref. [24]: $\mathrm{H}=0.11, \mathrm{C}=0.17, \mathrm{~N}=0.155, \mathrm{O}=0.152, \mathrm{Si}=0.21 \mathrm{~nm}$. In the last column, we list the markers used for plotting the points in Fig. 4.

\begin{tabular}{|c|c|c|c|c|c|c|}
\hline Name & Formula & $\kappa_{r}$ & $\tau_{\mathrm{PO}}(\mathrm{ns})$ & $\rho_{-}\left(\mathrm{nm}^{-3}\right)$ & $\tau^{\prime}(\mathrm{ns})$ & Marker \\
\hline$\alpha$ silica & $\mathrm{SiO}_{2}$ & $0.31 \pm 0.02$ & 0.27 & 234.6 & 0.24 & $\square$ \\
\hline Amorphous silica & $a-\mathrm{SiO}_{2}$ & $0.95 \pm 0.03$ & 1.59 & 234.6 & 1.4 & a \\
\hline Naphtalene & $\mathrm{C}_{10} \mathrm{H}_{8}$ & $0.82 \pm 0.07$ & 1.03 & 191.7 & 0.74 & $\diamond$ \\
\hline Acenaphthene & $\mathrm{C}_{12} \mathrm{H}_{10}$ & $0.59 \pm 0.05$ & 0.88 & 191.6 & 0.63 & - \\
\hline Polyethylene & $\left(\mathrm{C}_{2} \mathrm{H}_{4}\right)_{n}$ & 0.6 & 2.6 & 189.1 & 1.84 & $\Delta$ \\
\hline Atactic polypropylene & $\left(\mathrm{C}_{3} \mathrm{H}_{6}\right)_{n}$ & 0.66 & 2.66 & 189.1 & 1.88 & $\nabla$ \\
\hline Polyethylene terephthalate (PET) & $\left(\mathrm{C}_{10} \mathrm{H}_{8} \mathrm{O}_{4}\right)_{n}$ & 0.78 & 1.82 & 232.8 & 1.59 & $\star$ \\
\hline Butyl-PBD & $\mathrm{C}_{24} \mathrm{H}_{22} \mathrm{~N}_{2} \mathrm{O}$ & $0.88 \pm 0.11$ & 1.41 & 202.3 & 1.07 & $\Delta$ \\
\hline 2,5-diphenyloxazole (PPO) & $\mathrm{C}_{15} \mathrm{H}_{11} \mathrm{NO}$ & $0.78 \pm 0.04$ & 1.06 & 204.8 & 0.81 & $\boldsymbol{\gamma}$ \\
\hline 2,5-diphenyl-1,3,4-oxadiazole (PPD) & $\mathrm{C}_{14} \mathrm{H}_{10} \mathrm{~N}_{2} \mathrm{O}$ & $1.05 \pm 0.11$ & 1.22 & 218.6 & 1.00 & $\nabla$ \\
\hline
\end{tabular}




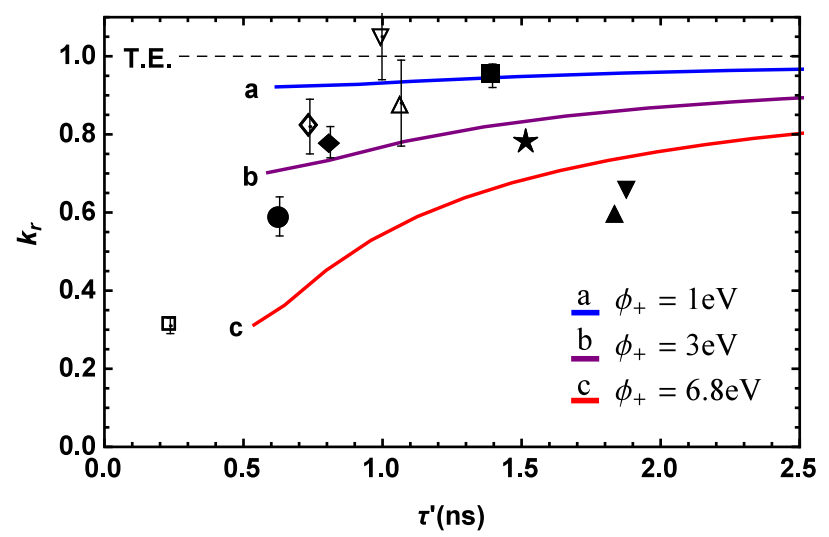

FIG. 4. Relationship between relative contact density $\kappa_{r}$ and $o$ Ps normalized lifetime $\tau^{\prime}$ for some materials. Full lines: theoretical results for three different values of the positron work function $\phi_{+}$. Markers: experimental data from Table I (only known errors are plotted). The reference dashed line indicates the result provided by the Tao-Eldrup model.

cannot be described with our model. On the contrary, for amorphous silica, we can predict a positron work function of $\phi_{+}=1 \pm 0.5 \mathrm{eV}$ and, from Fig. 3, a mean pore size of $1.2 \pm 0.5 \mathrm{~nm}$, consistent with existing data [19].

In conclusion, our theoretical model describes the properties of Ps confined in nanosized cavities and, in particular, justifies the well-known fact that the contact density is usually found to be well below the vacuum value. To our knowledge, this phenomenon was still missing a convincing explanation. The model considers different boundary conditions for the two particles (only the electron is strictly confined) and depends essentially on two parameters: the bulk positron work function and the pore size. Because these parameters are scarcely known in most materials, a comparison between theoretical findings and experimental data on contact densities and Ps lifetimes can be extremely useful for the determination of those quantities. Measurements of Ps lifetime in solids have a long story as a probe for guessing the size of internal cavities. We showed here that information on this quantity and on the positron work function can be extracted from independent measurements on Ps pickoff annihilation and contact density.

*Corresponding author. fabrizio.castelli@unimi.it

[1] A. Rich, Rev. Mod. Phys. 53, 127 (1981).

[2] S. Berko and H. N. Pendleton, Annu. Rev. Nucl. Part. Sci. 30, 543 (1980).
[3] A. Dupasquier, in Proceedings of the International School of Physics Enrico Fermi, Course 83, Varenna, Italy, edited by W. Brandt and A. Dupasquier (North-Holland, Amsterdam, 1983).

[4] V. I. Goldanskii, At Energy Rev. 6, 3 (1968).

[5] Y. C. Jean, in Proceedings of the International School of Physics Enrico Fermi, Course 125, Varenna, Italy, edited by A. Dupasquier and A. P. Mills, Jr. (IOS Press, Amsterdam, 1995).

[6] A. Dupasquier, P. DeNatale, and A. Rolando, Phys. Rev. B 43, 10036 (1991).

[7] G. Consolati and F. Quasso, Appl. Phys. A 52, 295 (1991).

[8] S. J. Tao, J. Chem. Phys. 56, 5499 (1972).

[9] M. Eldrup, D. Lightbody, and J. N. Sherwood, Chem. Phys. 63, 51 (1981).

[10] T. Goworek, K. Ciesielski, B. Jasińska, and J. Wawryszczuk, Chem. Phys. 230, 305 (1998).

[11] T. L. Dull, W. E. Frieze, D. W. Gidley, J. N. Sun, and A. F. Yee, J. Phys. Chem. B 105, 4657 (2001).

[12] W. Brandt, S. Berko, and W. W. Walker, Phys. Rev. 120, 1289 (1960).

[13] G. Consolati, F. Quasso, and D. Trezzi, PLoS One 9, e109937 (2014); K. Dvoyan, Physica (Amsterdam) 407B, 131 (2012).

[14] P. A. Sterne, L. Larrimore, P. Hastings, and A. L. R. Bug, Radiat. Phys. Chem. 68, 409 (2003).

[15] S. V. Stepanov, D. S. Zvezhinskiy, and V. M. Byakov, Mater. Sci. Forum 733, 7 (2013).

[16] M. J. Puska and R. M. Nieminen, Rev. Mod. Phys. 66, 841 (1994).

[17] N. Bouarissa and H. Aourag, Mater. Sci. Eng. B 34, 58 (1995).

[18] A. Rubaszek, in Proceedings of the International School of Physics Enrico Fermi, Course 125, Varenna, Italy, edited by A. Dupasquier and A. P. Mills, Jr. (IOS Press, Amsterdam, 1995).

[19] Y. Nagashima, Y. Morinaka, T. Kurihara, Y. Nagai, T. Hyodo, T. Shidara, and K. Nakahara, Phys. Rev. B 58, 12676 (1998).

[20] This is equivalent to considering the electron as completely surrounded by the material, i.e., in the center of an infinitely small cavity. This useful ansatz can be used because we need the wave function only in the half-space covered by the medium.

[21] D. Dutta, B. N. Ganguly, D. Gangopadhyay, T. Mukherjee, and B. Dutta-Roy, J. Phys. Condens. Matter 14, 7539 (2002).

[22] Note that this condition is more restrictive with respect to that defining the geometrical domain $\Omega(\vec{r}, \vec{R})$.

[23] R. A. Ferrell, Rev. Mod. Phys. 28, 308 (1956).

[24] A. Bondi, J. Phys. Chem. 68, 441 (1964); M. Mantina, A. C. Chamberlin, R. Valero, C. J. Cramer, and D. G. Truhlar, J. Phys. Chem. A 113, 5806 (2009). 